\title{
CARACTERIZACION ESPACIO-TEMPORAL DEL NICHO TROFICO DE LA FAUNA ICTICA ANDINA DEL RÍO MAULE, CHILE
}

\section{TROPHIC NICHE SPATIO-TEMPORAL CHARACTERIZATION OF THE ANDEAN ICHTHIC FAUNA AT THE MAULE RIVER, CHILE}

\author{
PatricioAcuña $^{1}$, Irma Vila ${ }^{1,}$ Rodrigo Pardo ${ }^{1} \&$ Sheila Comte $^{2}$ \\ ${ }^{1}$ Departamento de Ciencias Ecológicas, Facultad de Ciencias, Universidad de Chile, Las Palmeras 3425, Santiago. \\ Chile. Email: limnolog@uchile.cl \\ ${ }^{2}$ Facultad de Ecología y Ciencias Naturales, Universidad Nacional Andrés Bello, República 217, Santiago. Chile.
}

\begin{abstract}
RESUMEN
Los sistemas fluviales de la zona central de Chile presentan variaciones estacionales en: caudal, temperatura y conductividad. Esto ha influenciado a la fauna íctica nativa la que ha sido caracterizada como primitiva, representada por un bajo número de géneros y especies. La coexistencia de especies nativas e introducidas en el río Maule, Chile $\left(35^{\circ} 43^{\prime} \mathrm{S} ; 71^{\circ} 05^{\prime} \mathrm{W}\right)$, se remonta a comienzos del siglo XX, sistema en el cual las interacciones ecológicas entre estas especies no han sido suficientemente evaluadas. Para ello, se seleccionaron cuatro estaciones de trabajo en el sector andino del río Maule y sus tributarios, en dos periodos del año 1988. La fauna íctica se obtuvo realizando censo total, con un equipo de pesca eléctrica, analizándose el contenido estomacal de 142 ejemplares de Oncorhynchus mykiss, Basilichthys australis y Salmo trutta. La dieta de la mayoría de los peces analizados estuvo compuesta de organismos bentónicos, donde los taxa predominantes fueron Chironomidae, Ephemeroptera y Trichoptera. Se utilizó el índice de Horn para comparar la sobreposición de nicho trófico entre las diferentes especies, encontrándose un alto grado de sobreposición de nicho trófico en todas las estaciones y localidades. La sobreposición de nicho trófico y la diversidad bentónica se asociaron negativamente, lo que podría indicar cambios en la intensidad de las interacciones ecológicas causadas por cambios en el gradiente de recursos.
\end{abstract}

Palabras claves: Competencia interespecífica, fauna íctica introducida, sobreposición de nicho, río Maule.

\section{ABSTRACT}

Fluvial systems of central Chile show seasonal variations in: caudal, temperature and conductivity. This has influenced the ichthic native fauna, which has been characterized as primitive, with a low number of genera and species. The coexistence on native and exotic fishes in the Maule river, Chile $\left(35^{\circ} 43^{\prime} \mathrm{S} ; 71^{\circ} 05^{\prime} \mathrm{W}\right)$, is registered since the beginning of the XX century; system in which the ecological interactions between these species have not been adequately evaluated. For this, in between two different seasons of the year 1988, four different locations were selected in the Andean zone of the Maule river and its tributaries. The ichthic fauna was obtained using total census, with electrofishing equipment, stomach contents of 142 specimens of Oncorhynchus mykiss, Basilichthys australis and Salmo trutta were analized. The principal diet of the fishes analysed was basically benthic organisms, where Chironomidae, Ephemoroptera and Trichoptera were the predominant. Horn's index was used to compare trophic niche overlap among species, finding a high overlap index in all seasons and locations. Niche overlap and benthic diversity showed a negative relationship, this may indicate changes in the intensity of ecological interactions caused by changes in the gradient of resources.

KEYWORDS: Interespecific competition, exotic fishes, niche overlap, Maule river. 


\section{INTRODUCCION}

La introducción de peces exóticos ha traído generalmente resultados adversos para la ictiofauna nativa, por las alteraciones que producen en el hábitat, debido a los cambios en la estructura de la comunidad bentónica (Declerk et al. 2002), aumento de la turbiedad del agua, por la resuspensión del sedimento, introducción de parásitos y enfermedades (Fernando 1991) y principalmente la depredación y competencia por recursos (Arthington 1991).

La fauna íctica nativa de los sistemas fluviales de la zona centro sur de Chile se caracteriza por ser primitiva con un número bajo de géneros y especies, esto sería consecuencia de una gran adaptabilidad a un medio desfavorable (Campos 1970, Arratia 1981, Dyer 2000). Todas las especies nativas son depredadoras carnívoras, al igual que la mayor parte de la fauna íctica introducida de salmónidos, que se alimentan de presas bentónicas, principalmente crustáceos decápodos y larvas de insectos (Campos 1984, Campos et al. 1993, Ruiz et al. 1993, Habit 1998, Vila et al. 1999). Los peces carnívoros son generalmente depredadores visuales, y la presencia de una presa en su dieta depende de la disponibilidad de ésta, su detección y selección como alimento (Wootton 1998). Tanto la disponibilidad como la probabilidad de detección fluctúan considerablemente en las zonas ritrónicas de los ríos chilenos, debido a la alta variabilidad estacional en caudal, temperatura y material particulado que presentan estos sistemas, lo cual alteraría los patrones de interacciones ecológicas entre especies nativas y exóticas.

La cuenca del río Maule (VII región de Chile) es una de las cuatro más importantes del país.

Con una extensión de $20.295 \mathrm{~km}^{2}$ y una longitud de $240 \mathrm{~km}$, se ubica entre los $35^{\circ} 35^{\prime}$ y $35^{\circ} 50^{`} \mathrm{~S} ; 7^{\circ} 49^{`}$ y $71^{\circ} 04^{\top} \mathrm{W}$. El río Maule tiene un origen andino, se caracteriza por su escurrimiento torrencial y régimen mixto con crecidas pluviales en invierno, nivales en primavera y comienzos de verano, y estiaje pronunciado en otoño (Niemeyer \& Cereceda 1984).

La coexistencia de especies nativas e introducidas en el sector andino del río Maule se remonta a comienzos del siglo XX (De Buen 1959), período desde el cual las poblaciones de peces nativos se han visto afectadas por la presencia de las especies exóticas y cuyo efecto en las interacciones tróficas y su potencial variación estacional no ha sido suficientemente evaluado (Campos et al. 1998).

\section{MATERIALES Y METODOS}

Se seleccionaron cuatro localidades de trabajo ubicadas en el sector andino de la cuenca del río Maule, aguas arriba del embalse Colbún, situadas en: el río Claro (A), estero Suiza (B), río Maule (C), río Melado (D), donde las localidades A, B y D son tributarios del río Maule. Se realizaron dos campañas en mayo y diciembre de 1988, correspondientes a otoño y fines de primavera respectivamente. La fauna íctica se obtuvo realizando censo total, cercando con redes agalleras segmentos de río de aproximadamente 20 metros de largo, capturando la totalidad de los peces del lugar con un equipo de pesca eléctrica portátil marca Coffelt. Los ejemplares capturados fueron identificados, registrándose longitud total con precisión de $0.1 \mathrm{~mm}$, peso total y eviscerado con precisión de $0.1 \mathrm{~g}$. Los estómagos se preservaron en formalina al $10 \%$, para el posterior recuento e identificación de presas, utilizando para esto una lupa estereoscópica. Las presas se identificaron según Merritt \& Cummins (1978) y Lopretto \& Tell (1995) hasta nivel de orden, excepto en Diptera, donde se identificó la familia Chironomidae. Además, en cada campaña y localidad se obtuvieron muestras de fauna bentónica mediante red Surber de $0.09 \mathrm{~m}^{2}$ por duplicado. Las muestras fueron fijadas en formalina al 10\% para su posterior identificación taxonómica y determinación de abundancia, riqueza y diversidad según Simpson (Krebs 1999).

Los datos de contenido estomacal de los peces se agruparon por: especie, campaña, localidad y longitud de clase en intervalos de $5.0 \mathrm{~cm}$, calculándose la proporción de cada presa en su dieta. Se estimó la sobreposición de nicho trófico interespecífico, mediante el índice de Horn (Krebs 1999), entre categorías de longitud para cada localidad en ambas campañas. Se calculó este índice para todas las localidades en las dos campañas, excepto la localidad B en diciembre, debido a que sólo se encontróOncorhynchus mykiss (Walbaum). El índice de Horn presenta ventajas frente a otras medidas de sobreposición de nicho, debido a su menor sesgo en su estimación (Krebs 1999). Con el fin de evaluar posibles diferencias en la sobreposicion de nicho entre localidades y cambios temporales en cada localidad, se realizó análisis de varianza no paramétrico de Kruskal-Wallis. Además, se realizaron correlaciones no paramétricas de Kendall entre los índices bentónicos obtenidos y la sobreposición de nicho trófico para ambas campañas. 


\section{RESULTADOS}

Durante el período de estudio, la fauna íctica en la zona cordillerana del río Maule estuvo conformada por las especies Oncorhyhus mykiss, Basilichthys australis Eigenmann, 1927, Salmo trutta Linnaeus, 1758 y Trichomycterus sp. 96 ejemplares de O. mykiss, 40 de $B$. australis y 6 de $S$. trutta presentaron contenido estomacal, durante las campañas de otoño y primavera.

La fauna bentónica varió temporalmente, en la campaña de mayo se encontraron Coleoptera, Tricoptera y Ephemeroptera como los insectos más abundantes en las cuatro localidades. Chironomidae y Diptera presentaron menor abundancia en todas las localidades. La mayor riqueza se registró en diciembre, con nueve taxa. Durante este mes, la abundancia de la fauna bentónica cambió en comparación con la campaña anterior, aumentó Chironomidae y Diptera en todas las localidades y disminuyó Ephemeroptera y Coleoptera.

Los organismos bentónicos son las presas que componen en su mayoría la dieta de los peces analizados, con una fuerte predominancia de Chironomidae, que representó el $57 \%$ del total del contenido estomacal en B. australis y el $31 \%$ en O. mykiss. La segunda presa de mayor importancia fue Ephemeroptera con $28 \%$ y $21 \%$ para O. mykiss y B. australis, respectivamente. En $S$. trutta las presas más abundantes fueron Tricoptera con $34 \%$ y Chironomidae con $21 \%$. O. mykiss es la única especie, de las analizadas, que exhibió peces como una de sus presas. Además, de insectos del Orden Hymenoptera, los cuales corresponden a fauna de deriva.

La sobreposición de nicho trófico, calculado como el índice de Horn, presentó su valor mínimo en la localidad $\mathrm{C}$ durante la campaña de mayo (0.587) y el máximo en esta misma localidad en la campaña de diciembre (0.901). Se encontraron diferencias significativas entre los índices de Horn obtenidos para cada localidad en las dos campañas (KruskalWallis; $\mathrm{p}<0.001)$. Al analizar los datos temporalmente en cada localidad, sólo en la localidad C se encontraron diferencias entre las dos campañas (Kruskal-Wallis; p <0.001) siendo la sobreposición de nicho trófico mayor en diciembre (Fig. 1). En la localidad B para la campaña de diciembre no se calculó este índice debido a que sólo se capturaron ejemplares de O. mykiss. Al analizar los datos espacialmente dentro de cada campaña, sólo se encontraron diferencias en la campaña de diciembre, entre las localidades C y D (Kruskal-Wallis; $\mathrm{p}<0.005)$. El índice de Horn se correlacionó significativamente con la diversidad bentónica en las campañas de otoño $(=-0.35 ; \mathrm{p}<0.05)$ y primavera ( $=-0.48 ; \mathrm{p}<0.001)$. Por otro lado, no se observaron asociaciones significativas entre el índice de Horn y la riqueza o la abundancia bentónica.

\section{DISCUSION}

En la actualidad existe escasa información sobre los posibles cambios en las tramas tróficas, causados por la introducción de salmónidos en Chile. La información de la fauna íctica habitante en el sector andino del río Maule revela la introducción de trucha arco iris $O$. mykiss previo a la década de los años cincuenta (De Buen 1959). Las características de estos ríos andinos favorecería a los salmónidos introducidos, que presentarían ventajas adaptativas dado que su lugar de origen corresponde a ríos de tipo ritrónico, son seleccionadas en los cultivos artificiales, poseen conducta agresiva y alcanzan tamaños corporales mayores comparadas con las especies nativas (Campos et al. 1998) encontradas en el área, situación que podría verse reflejada en la presencia única de $O$. mykiss en la localidad B para el período de primavera.

Los índices de sobreposición de nicho obtenidos como series de tiempo, en el mismo lugar, pueden ser útiles estimadores de posibles interacciones interespecíficas, como la competencia (Núñez et al. 1989). La magnitud de estas interacciones puede verse alterada, por la disponibilidad del recurso en cuestión, como se observa en otoño y primavera, con la asociación negativa entre diversidad de presas disponibles y sobreposición de nicho trófico. La sobreposición de nicho trófico en el río Maule disminuyó a mayor diversidad de recurso, esto podría atribuirse a que al aumentar la diversidad de la oferta, el gradiente de recurso se amplía, y en situaciones de alta competencia se esperaría que las especies se segregaran más, ya que el ambiente estaría ofreciendo nuevos recursos no utilizados por otras especies. Por el contrario a menor diversidad de oferta el gradiente de recursos disminuye, obligando a 
las especies a aumentar su sobreposición de nicho el caso extremo ocurriría cuando el recurso es sólo una especie, donde la sobreposición sería total. Debido al menor tamaño de la fauna íctica nativa y la piscivoría que presentan los salmónidos, en perío- dos de baja diversidad de recursos, en los que se vería aumentada la magnitud de las interacciones competitivas, se esperaría un efecto asimétrico entre las especies nativas e introducidas que afectaría negativamente a nuestra fauna nativa.

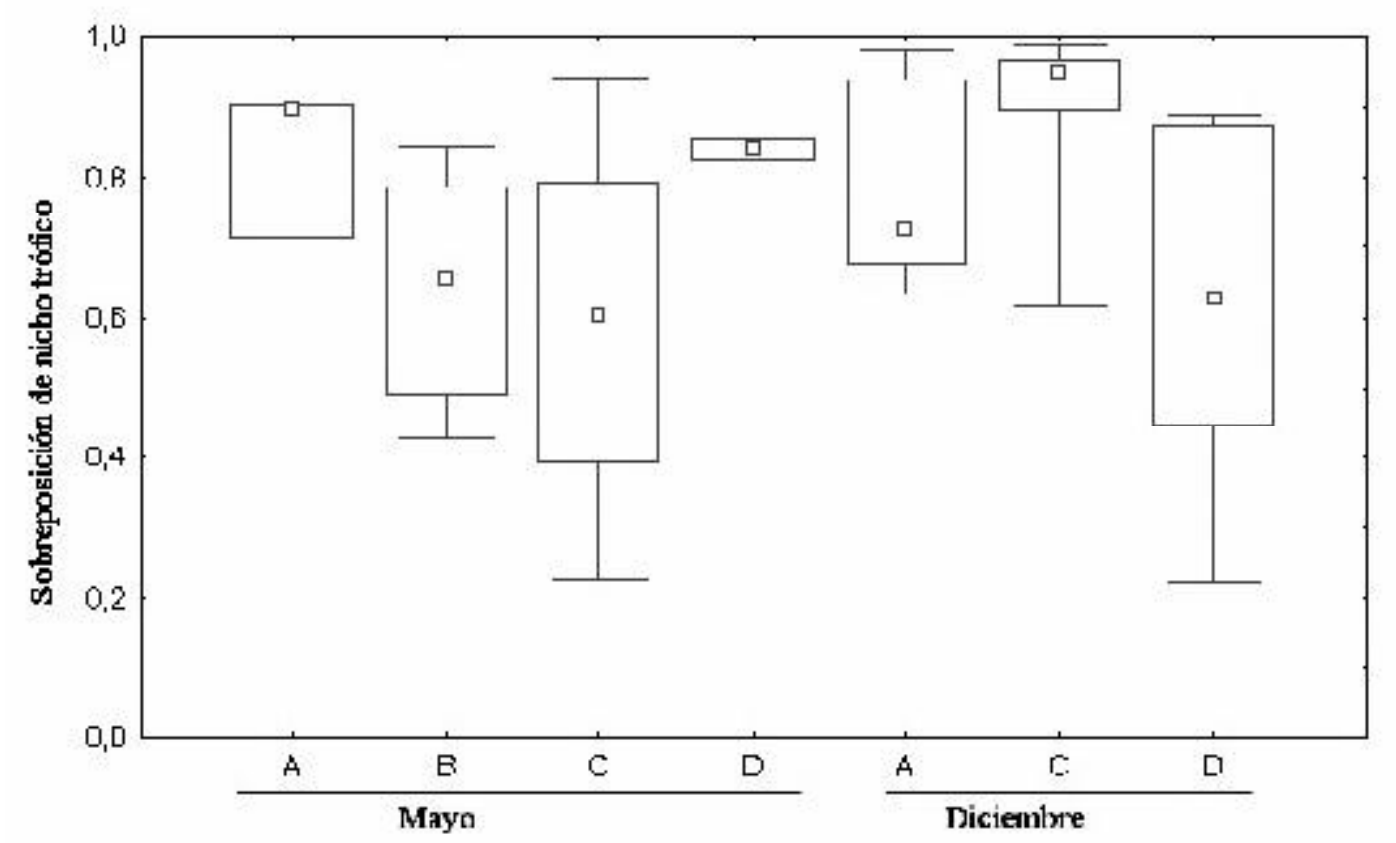

FIguRa 1. Indice de Horn para las localidades: (A) río Claro, (B) estero Suiza, (C) río Maule y (D) río Melado, en las campañas de mayo y diciembre. Los puntos indican la mediana, las cajas desviación estándar y las líneas señalan los valores máximos y mínimos.

FiguRe 1. Horn`s index for locations: (A) río Claro, (B) estero Suiza, (C) río Maule y (D) río Melado, at may and december campaigns. Points are the median value, box are the standard deviation and lines represent maximum and minimum values.

\section{AGRADECIMIENTOS}

Rodrigo Pardo es beneficiario de una beca MECESUP UCO- 0214.

\section{BIBLIOGRAFIA}

Arratia, G. 1981. Géneros de peces de aguas continentales de Chile. Publicación Ocasional Mus. Nac. Hist. Nat. 34:3-108.

Arthington, A.H. 1991. Ecological and genetic impacts of introduced and translocated freshwater fishes in Australia. Can. J. Fish Aquat. Sci. 48 (Suppl.1):33-43.

Campos, H. 1970. Introducción de especies exóticas y su relación con los peces de agua dulce de Chile. Not.
Mens. Mus. Nac. Hist. Nat. 162:3-9.

Campos, H. 1984. Productividad íctica de ríos y lagos araucanos. En: Vila, I \& E. Fagetti, (eds). Trabajos presentados al Taller Internacional sobre ecología y manejo de peces en lagos y embalses. Santiago, Chile, 5-10 de noviembre de 1984. COPESCAL Doc. Téc. (4):237 pp.

Campos, H., J. F. Gavilán, F. Alay \& V. Ruiz. 1993. Comunidad íctica de la hoya hidrográfica del río Bío Bío. En: Evaluación de la calidad del agua y ecología del sistema limnético y fluvial del río Bío Bío. Monografía EULA 12:249-278.

Campos, H., G. Dazarola, B. D yer, L. Fuentes, J. F. Gavilán, L. Huaquín, G. Martínez, R. Meléndez, G. Pequeño, F. Ponce, V. H. Ruiz, W. Sielfeld, D. Soto, R. Vega \& I. Vila. 1998. Categorías de conservación de peces nativos de aguas continentales de Chile. Bol. Mus. Nac. Hist. Nat. 47:101-122. 
De Buen, F. 1959. Los peces exóticos en las aguas dulces de Chile. Inv. Zool. Chilenas. 5:103-137.

Declerck, S., G. Louette, T. De Bie \& L. De Meester. 2002. Patterns of diet overlap between populations of non-indigenous and native fishes in shallow ponds. J. Fish Biol. 61:1182-1197.

Dyer, B. 2000. Systematic review and biogeography of the freshwater fishes of Chile. Estud. Oceanol. 19:77-98.

Fernando, C. H. 1991. Impacts of fish introductions in tropical Asia and America. Can. J. Fish Aquat. Sci. 48 (Suppl.1):24-32.

Haвıт, E. 1998. Análisis de la dieta de Percilia gillissi (Pisces:Perciliidae) en poblaciones de río y canales de riego (cuenca del Itata, VIII Región). Theoria. 7:33-46.

Krebs, C. 1999. Ecological Metodology. Second Edition. Benjamin/Cummings, Addison Wesley, Menlo Park, California. 620 pp.

Lopretto, E. \& G. Tell. 1995. Ecosistemas de Aguas Continentales. Tomo III. Ediciones Sur. La Plata, Argentina. $1401 \mathrm{pp}$.
Merritt, R. W. \& K.W. Cummins. 1978. An Introduction to the Aquatic Insects of North America. Kendall/Hunt Publishing. Iowa. $441 \mathrm{pp}$.

Niemeyer, H. \& P. Cereceda. 1984. Hidrografía. Geografía de Chile. Tomo VIII. Editado por Instituto Geográfico Militar. Chile. 320 pp.

Núñez, H., P. A. Marquet, R. G. M edel \& F. Jaksic. 1989. Niche relationships between two sympatric Liolaemus lizards in a fluctuating environment: the "lean" versus "feast" scenario. J. Herpetol. 23:22-28

Ruiz, V. H., M. T.López, H. I. M oyano \& M. M archant. 1993. Ictiología del alto Bío Bío: aspectos taxonómicos, alimentarios, reproductivos y ecológicos con una discusión sobre la hoya. Gayana Zool. 57(1):77-88.

Vila, I., L. Fuentes \& M. Contreras. 1999. Peces límnicos de Chile. Bol. Mus. Hist. Nat. 48:6175.

Wootton, R. J. 1998. Ecology of teleost fishes. Second edition. Kluwer Academic Publishers. 386 pp. 